\title{
(a) OPEN ACCESS \\ Relationship between socioeconomic status and incidence of out-of-hospital cardiac arrest is dependent on age
}

\author{
Martin Jonsson (10, ${ }^{1}$ Petter Ljungman, ${ }^{2}$ Juho Härkönen (10, ${ }^{3,4}$ Ben Van Nieuwenhuizen, ${ }^{5}$ \\ Sidsel Møller, ${ }^{6}$ Mattias Ringh, ${ }^{1}$ Per Nordberg ${ }^{1}$, for the ESCAPE-NET investigators
}

\begin{abstract}
- Additional material is published online only. To view please visit the journal online (http://dx.doi.org/10.1136/ jech-2019-213296).

${ }^{1}$ Center for Resusctitation Science, Department of Medicine, Solna, Karolinska Institutet, Stockholm, Sweden ${ }^{2}$ Institute of Environmental Medicine, Karolinska Institutet, Stockholm, Sweden ${ }^{3}$ Department of Political and Social Sciences, European University Institute, Florence, Italy

${ }^{4}$ Department of Sociology, Stockholm University,

Stockholm, Sweden ${ }^{5}$ Department of Public Health, Amsterdam UMC, Amsterdam, the Netherlands

${ }^{6}$ Department of Cardiology, Gentofte Hospital, Copenhagen, Denmark
\end{abstract}

Correspondence to Martin Jonsson, Center for Resuscitation Science, Department of Medicine, Karolinska Institutet, Stockholm 118 83, Sweden; martin.k.jonsson@ki.se

Received 27 October 2019 Revised 2 April 2020 Accepted 21 April 2020

\section{Check for updates}

(C) Author(s) (or their employer(s)) 2020. Re-use permitted under CC BY-NC. No commercial re-use. See rights and permissions. Published by BMJ.

To cite: Jonsson M, Ljungman P, Härkönen J, et al. J Epidemiol Community Health 2020;74:726-731.

\begin{abstract}
Background The association between socioeconomic status (SES) and incidence of out-of-hospital cardiac arrest (OHCA) is not fully understood. The aim of this study was to see if area-level socioeconomic differences, measured in terms of area-level income and education, are associated with the incidence of OHCA, and if this relationship is dependent on age.

Methods We included OHCAs that occurred in Stockholm County between the 1st of January 2006 and the 31 st of December 2017, the victims being confirmed residents ( $n=10574)$. We linked the home address to a matching neighbourhood (base unit) via available socioeconomic and demographic information.

Socioeconomic variables and incidence rates were assessed by using cross-sectional values at the end of each year. We used zero-inflated negative binomial regression to calculate incidence rate ratios (IRRs).

Results Among 1349 areas with complete SES
\end{abstract} information, 10503 OHCAs occurred between 2006 and 2017. The IRR in the highest versus the lowest SES area was $0.61(0.50-0.75)$ among persons in the $0-44$ age group. Among patients in the 45-64 age group, the corresponding IRR was 0.55 (0.47-0.65). The highest SES areas versus the lowest showed an IRR of 0.59 $(0.50-0.70)$ in the 65-74 age group. In the two highest age groups, no significant association was seen (75-84 age group: 0.93 (0.80-1.08); 85+ age group: 1.05 (0.84-1.23)). Similar crude patterns were seen among both men and women.

Conclusions Areas characterised by high SES showed a significantly lower incidence of OHCA. This relationship was seen up to the age of 75 , after which the relationship disappeared, suggesting a levelling effect.

\section{INTRODUCTION}

There is a link between socioeconomic status (SES) and cardiovascular health outcomes, including allcause mortality, prevalence of risk factors and incidence rates of cardiovascular diseases such as myocardial infarction and stroke. ${ }^{2}$ Out-of-hospital cardiac arrest (OHCA) is a major public health concern, annually affecting more than 350000 persons in the $\mathrm{US}^{3}$ and 300000 in Europe, ${ }^{4}$ with a mortality rate of around $90 \%{ }^{4}$

The results of several observational studies have shown associations between different measurements of SES and incidence rates of OHCA in North America, ${ }^{5-7}$ Asia $^{8}{ }^{9}$ Europe $^{10-12}$ and Australia. ${ }^{13}$ In a recent review, these results were corroborated, and it was concluded that low SES areas generally showed a higher incidence of OHCA. ${ }^{14}$ It is important to further identify factors that lead to a high incidence of OHCA in order to be able to guide health interventions such as placement of public automated external defibrillators (AEDs) and to improve cardiopulmonary resuscitation (CPR) skills in those high-risk areas.

Two studies from North America revealed a stronger association between area-level SES and incidence of OHCA among people under the age of 65 years, compared with patients aged $\geq 65$ years. ${ }^{56}$ These results suggest that the relationship between SES and incidence of OHCA may differ depending on age.

To our knowledge, no study has been carried out to investigate the association between area-level SES and incidence of OHCA in a range of age groups. A better understanding of how age interacts with SES is of importance in order to find the most suitable locations for public health interventions.

In the present study, we compared the incidence rates of OHCA in neighbourhood areas (base units) within the Stockholm region, Sweden, in relation to SES, defined in terms of income and education. The aim was to determine if area-level SES was associated with the incidence of OHCA and how this possible relationship is related to age.

\section{METHODS}

\section{Study design and ethics}

The study was a retrospective observational study using area-level data from Statistics Sweden and OHCA data from the Swedish Register or Cardiopulmonary Resuscitation (SRCR). The study was approved by the regional ethics review board (identification number 2017/714-31/5).

\section{Study setting}

The Stockholm region covers $6517 \mathrm{~km}^{2}$ and included 1918104 inhabitants in 2006, which increased to 2308143 inhabitants in 2017. The crude incidence rate of cardiac arrest is $\approx 900-1100$ per year. The Stockholm region is divided into 1418 neighbourhoods where summary statistics are available. The median population size among base units over the study period was 1054 (Q1=301, Q3 =2117).

\section{Study population}

All OHCAs treated by emergency medical services (EMS) in the region of Stockholm and registered in 
the Swedish Register for Cardiopulmonary Resuscitation (SRCR) were included in the study. The SRCR is a national quality register supported by the Swedish Association of Local Authorities and Regions. The register was created at the beginning of the 1990s and is used to measure the overall quality of OHCA treatment in Sweden. All EMS in Sweden report to the registry, following an Utstein-based template. ${ }^{10}$ Patients of unknown identity and individuals who resided outside Stockholm County were excluded.

\section{Sociodemographic measurements}

Area-level SES was defined by income and education, aggregated by each of the 1418 base units in the Stockholm region. We used median income from employment and the proportion of inhabitants (between the ages of 16 and 76 years) with three or more years of university-level education, similar to a bachelor-level degree or higher. To categorise the areas by SES, median values (eg, income) were calculated in a cross-sectional manner for each year and later divided by the number of years in which data were available (for changes over time, please see figures $\mathrm{S} 5$ and S6). Quintiles of both income and education were used to create an SES index, with a minimum score of two (lowest quintile in both income and education) and a maximum score of 10 (highest quintile in both income and education). The index was categorised into five groups (low SES 2-3 points; medium-low SES 4-5; medium SES 6-7; medium-high SES $8-9$ and high SES 10).

The coordinates of the home address of the person that suffered an OHCA (regardless of where the OHCA occurred) were linked to the base-unit areas using geographical information systems (R package; SP). Demographic variables for each base unit were acquired from the Stockholm regional area database with data from Statistics Sweden. ${ }^{15}$ The database is operated by the region of Stockholm and contains demographic data on an area level.

\section{Spatial autocorrelation}

To check for spatial autocorrelation of OHCA incidence, we used Moran's I. The non-significant $\mathrm{p}$ value of 0.405 suggested that this was not a problem.

\section{Statistical methods}

To assess the associations between area-level SES and incidence of OHCA, we calculated our results in terms of crude incidence per 100000 person-years and incidence rate ratios (IRRs). The IRRs were calculated using a zero-inflated negative binomial (ZINB) regression model. ${ }^{16}$ We chose this analytical method, given the excess zeros and overdispersion of the data. The ZINB model was compared with quasi-Poisson regression, zero-inflated Poisson regression and negative binomial regression using Vuong's nonnested hypothesis test. All ZINB analyses were adjusted for the proportion of people of foreign background (defined as either born or both parents born outside the Nordic countries) and unemployment rate (percentage unemployed for more than 6 months among persons aged 18-64). This resulted in the following ZINB model: Incidence $\sim$ SES + foreign + unemployment | population.

In the models where all age groups were included, additional adjustment was made for the proportion of the population over the age of 65 years. All regression equations are included in the supplementary appendix.
To handle the problem of influential cases, we excluded areas of high incidence by using the $1.5 \mathrm{IQR}$ rule. ZINB regression analyses were performed for the following age groups: $0-44,45-64,65-74,75-84$ and 85 and older, and all age groups together. These analyses were repeated for men and women separately, resulting in a total of 18 regression analyses. We categorised age in the above-specified groups in order to have enough OHCAs in each age group. As the incidence is much lower among younger persons, the youngest age group needed to cover more years than the others. The variable used for discrimination of excess zeros was the total population in each age group. We used incidence per 100000 person-years (per age group) as the outcome measure in all regression analyses. All tests were two sided, and $\mathrm{p}$ values below 0.05 were considered statistically significant. All statistical procedures were performed with $\mathrm{R}$ statistical software, version 3.6.2 for Mac OSx (R Foundation for Statistical Computing, 2019).

\section{RESULTS}

Between 1 January 2006 and 31 December 2017, a total of 11784 EMS-treated OHCAs occurred in Stockholm. Of these, 10574 were confirmed residents of the Stockholm region. The 1210 excluded patients were of unknown identity, were living outside Stockholm or were residents of another country. Among the 1418 base units, 69 had missing information on either income or education and were therefore excluded from the analysis.

\section{Demographic variables categorised by SES (table 1)}

Areas of low SES (categorised according to income and education) had higher proportions of people with a foreign background. In the lowest SES area, the proportion of the population with a foreign background was $55.6 \%$, decreasing to $16.1 \%$ in the highest SES area. Notable differences were seen among the proportions unemployed for more than 6 months, with $3.5 \%$ in the lowest SES areas compared with $0.5 \%$ in the high SES areas. The proportion of men was similar in all areas (49\%-50\%). The AED density (per $\mathrm{km}^{2}$ ) was much higher in the higher SES areas $\left(1.59,4.52\right.$ per $\left.\mathrm{km}^{2}\right)$ compared with the lower ones $\left(0.26,0.20\right.$ per $\left.\mathrm{km}^{2}\right)$. For further details, see table 1 .

\section{Total incidence rate by SES quintile (figure 1)}

The crude incidence rate was 52 per 100000 person-years in the lowest and second-lowest SES areas. The medium SES areas showed an incidence rate of 38 per 100000 person-years. In the two highest SES areas, the incidence rates dropped to 34 (medium-high SES) and 26 (high SES) per 100000 personyears (figure 1). After adjustment for the proportion of the population with a foreign background, unemployed people and the proportion older than 65 years, there was a negative gradient in IRRs with increasing level of SES. The highest SES areas showed an IRR of 0.63 (95\% CI 0.56 to 0.71 ).

\section{Proportions of OHCAs and population according to age (figure 2)} Age group 0-44 years included $60 \%$ of the total population and $10 \%$ of the cardiac arrests. Corresponding figures for the other age groups were: $45-64$ age group, population $24 \%$, OHCAs $24 \% ; 65-74$ age group, population $9 \%$, OHCAs $23 \% ; 75-84$ age group, population $5 \%$, OHCAs $24 \%$; $85+$ age group, population $2 \%$, OHCAs $19 \%$. 
Table 1 Characteristics of areas by socioeconomic status

\begin{tabular}{|c|c|c|c|c|c|}
\hline & Low & $\begin{array}{l}\text { Medium- } \\
\text { low }\end{array}$ & Medium & $\begin{array}{l}\text { Medium- } \\
\text { high }\end{array}$ & High \\
\hline Number of areas & 294 & 302 & 324 & 274 & 155 \\
\hline $\begin{array}{l}\text { Proportion with } \\
\text { university education }\end{array}$ & $12.2 \%$ & $18.4 \%$ & $27 \%$ & $36.3 \%$ & $46.1 \%$ \\
\hline Median income* & 20140 & 26449 & 29841 & 33266 & 37840 \\
\hline $\begin{array}{l}\text { Proportion with } \\
\text { foreign background }\end{array}$ & $41.2 \%$ & $22.9 \%$ & $17.1 \%$ & $13.1 \%$ & $12.8 \%$ \\
\hline $\begin{array}{l}\text { Proportion } \\
\text { unemployed } \\
\text { (>6 months among } \\
\text { 18- to 64-year-olds) }\end{array}$ & $3.5 \%$ & $1.6 \%$ & $1.0 \%$ & $0.8 \%$ & $0.5 \%$ \\
\hline $\begin{array}{l}\text { Proportion over } \\
65 \text { years }\end{array}$ & $14.4 \%$ & $17.6 \%$ & $15.4 \%$ & $14.5 \%$ & $13.2 \%$ \\
\hline Proportion of men & $50.1 \%$ & $49.3 \%$ & $49.6 \%$ & $49.2 \%$ & $49.4 \%$ \\
\hline $\begin{array}{l}\text { Total } \\
\text { person-years }\end{array}$ & 5485825 & 4977189 & 5884484 & 5912268 & 3031962 \\
\hline Total OHCAs & 2867 & 2577 & 2248 & 2028 & 783 \\
\hline OHCA age $0-44$ & 346 & 234 & 198 & 180 & 66 \\
\hline OHCA age $45-64$ & 769 & 614 & 495 & 448 & 168 \\
\hline OHCA age $65-74$ & 714 & 616 & 530 & 428 & 169 \\
\hline OHCA age $75-84$ & 644 & 640 & 572 & 505 & 207 \\
\hline OHCA age $85+$ & 389 & 470 & 453 & 465 & 171 \\
\hline $\begin{array}{l}\text { Crude incidence rate } \\
\text { per } 100000\end{array}$ & 52 & 52 & 38 & 34 & 26 \\
\hline Number of AEDs & 572 & 555 & 639 & 572 & 408 \\
\hline Area in $\mathrm{km}^{2}$ & 2186 & 2793 & 1015 & 360 & 90 \\
\hline $\mathrm{AEDs} / \mathrm{km}^{2}$ & 0.26 & 0.20 & 0.63 & 1.59 & 4.52 \\
\hline
\end{tabular}

*Euros calculated from Swedish currency using the value of the euro on the 31st of December 2010.

AED, automated external defibrillator; OHCA, out-of-hospital cardiac arrest.
Incidence by age group (figure 3 )

Among patients in the age group $0-44$ years, the incidence of OHCA was 10 per 100000 person-years in the lowest SES areas compared with 5 per 100000 in the highest SES areas. The corresponding IRR was 0.56 (95\% CI 0.45 to 0.70 ) in the highest SES areas compared with the lowest SES areas. In the age group 45-64 years, there was a similar negative gradient with increasing SES. The crude incidence was 61 per 100000 person-years in the lowest SES areas compared with 23 per 100000 in the highest SES areas. The corresponding IRR was 0.53 (95\% CI 0.45 to 0.62 ). The negative gradient was present among the 65-74 age group as well, with crude rates of 165 per 100000 person-years in the lowest SES areas and 69 per 100000 in the highest SES areas. The IRR in the highest SES areas was 0.59 (95\% CI 0.49 to 0.70$)$ compared with the lowest. The negative trend disappeared in the 75-84 age group, with a crude incidence rate of 254 in the lowest SES areas compared with 195 in the highest SES areas. The IRR was 0.87 (95\% CI 0.74 to 1.02) in the highest SES areas versus the lowest SES areas. Among the oldest group $(85+)$, the results were similar, with no trend by SES. All IRRs presented above were adjusted for the proportion of those with a foreign background and unemployment rate.

\section{Incidence by sex and age group}

Among men (figures S1), the negative trend was present from the $0-44$ age group up to the 65-74 age group. In the 75-84 age group, the negative gradient was weaker, and among the oldest men $(85+)$ the trend disappeared. Among women (Figure S2), the negative gradient could be seen up to the 75-84 age group in crude rates. In the youngest age group (0-44), the IRRs followed a strong negative gradient in relation to SES (IRR 0.31, 95\% CI 0.19 to 0.49 ). In the other age groups, the trend was much weaker and could no longer be seen in age groups 75-84 and

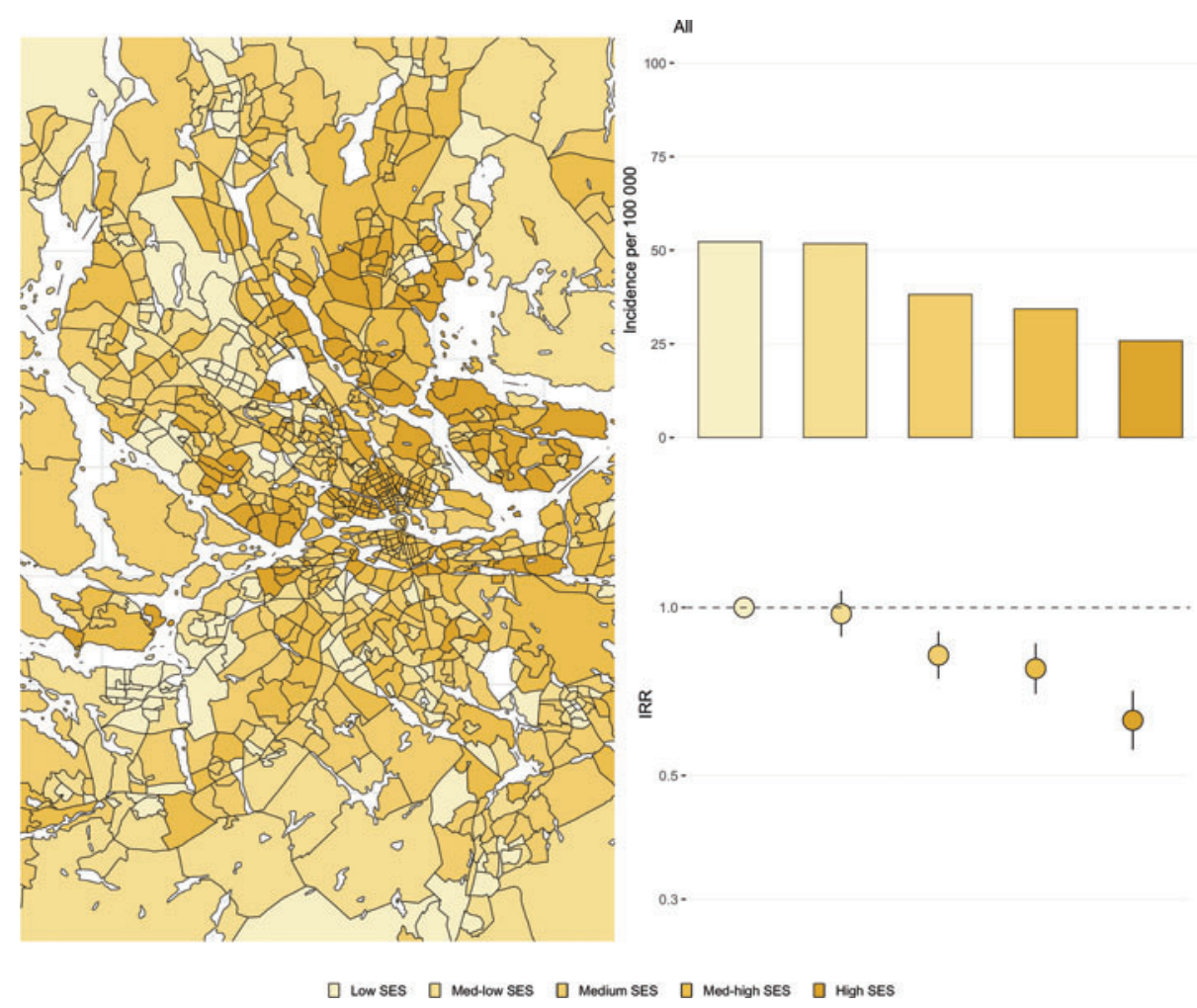

Figure 1 Map of neighbourhoods (base units) in the Stockholm region. Top right: Crude incidence per 100000 person-years by area-level socioeconomic status (SES). Bottom right: Incidence rate ratios (adjusted for proportion with foreign background, unemployment rate and proportion over 65 years of age) by area-level. 


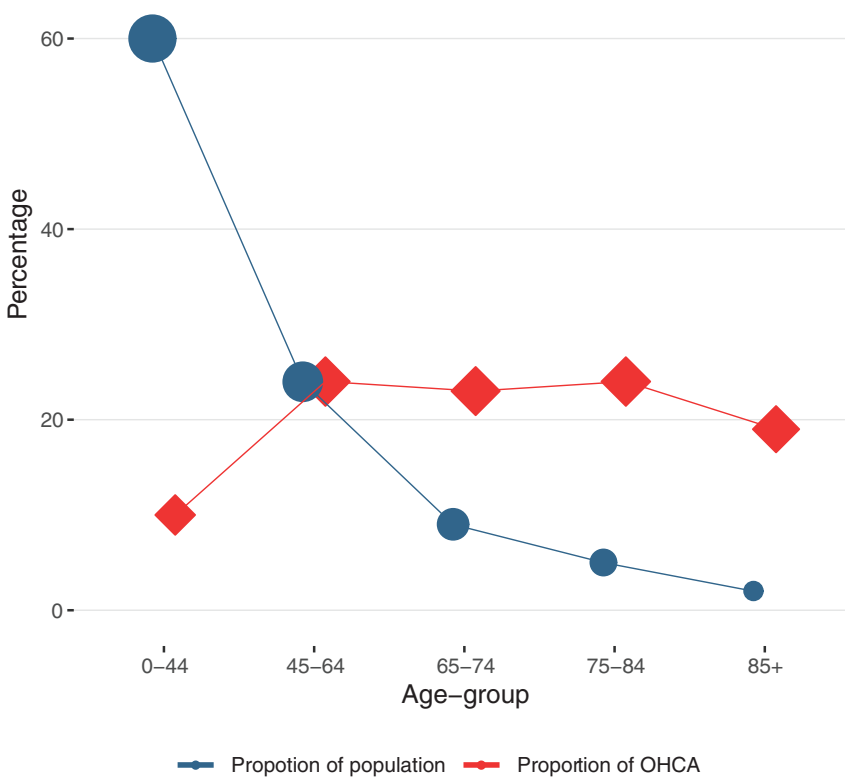

Figure 2 Proportion of population and proportion of OHCA cases categorised by age group. OHCA, out-of-hospital cardiac arrest.

$85+$. As above, the IRRs were adjusted for the proportion of those with a foreign background and unemployment rate.

\section{Sensitivity analyses}

In addition to the analyses presented, we conducted separate analyses after dividing the areas into groups by the percentage of those with a foreign background (Tables S1-S3). The negative association was present in all analyses $(<10 \%, 10-19 \%$ and $\geq 20 \%)$. All analyses were repeated using income (Figure S3) and education (Figure S4) alone as SES measures. These analyses produced similar results as those included here (see supplementary appendix).

\section{DISCUSSION}

The main finding in the present study was a significantly higher incidence of OHCA in areas characterised by low versus high SES. This relationship seems to be dependent on age, as no association was seen in patients over 75 years of age. The relationship between SES and OHCA incidence appeared to be stronger among men versus women.

Although the SES differences tended to be weaker in the older age groups, there appeared to be a strong correlation between SES and incidence of OHCA in most cases. These results may have several important clinical implications. First, they could be used to guide AED programme implementation. For an AED programme to be successful, it is crucial that the defibrillators are placed where there is a high probability of OHCA occurrence. The results suggest a much lower AED density in areas categorised by low SES, which stresses the importance of this. Second, other interventions such as programmes aimed at increasing awareness of how to act in an emergency situation, and CPR training, could be directed towards low SES areas.

Our results are consistent with those in previous work concerning SES and OHCA incidence, showing a higher incidence in low SES areas. ${ }^{568-111314}$ Reinier et $^{6 l^{5}}{ }^{6}$ reported similar results in the US, with a stronger relationship between SES and OHCA incidence among younger persons. The authors proposed that this may be a result of access to healthcare, as Medicare is available from 65 years of age. A recent study by Stecker et $a l^{17}$ showed a significant decrease of OHCA incidence among the middle-aged population after implementation of the Affordable Care Act in the US, which gives some support to this explanation. In contrast to the US, Sweden, along with other countries such as South Korea, ${ }^{8}$ Denmark ${ }^{10}$ and the UK, ${ }^{12}$ has universal healthcare coverage, so the explanation that access to healthcare would attenuate the SES gradient is not likely to apply to the same extent. Our results suggest that even with a universal healthcare system, significant differences between SES areas exist.

The attenuated effect of SES in successively older age groups is consistent with the 'age-as-leveler' hypothesis, ${ }^{18}$ which suggests
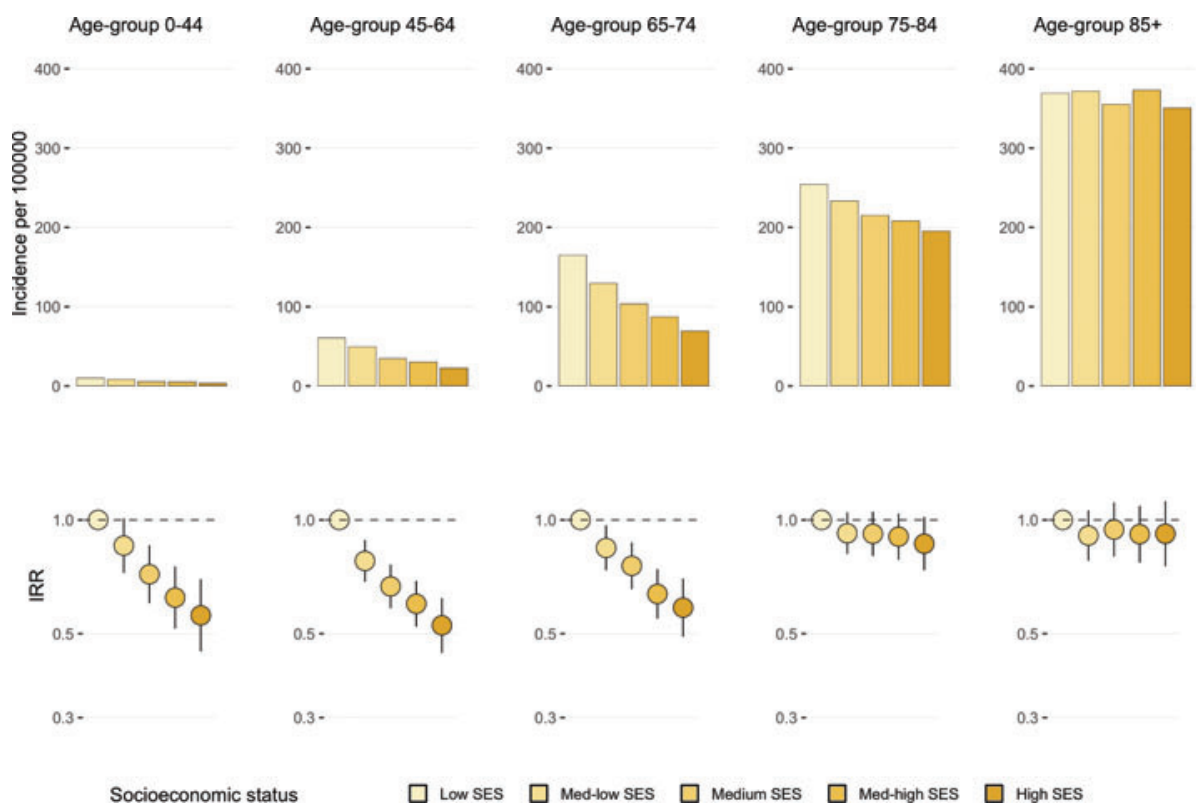

Figure 3 Crude incidence rates by area-level socioeconomic status and age group (top row) and corresponding incidence rate ratios (adjusted for proportion of population with foreign background and unemployment rate) by area-level socioeconomic status (SES) and age group (bottom row). 
that socioeconomic differences diminish with older age. One of the possible and perhaps most probable ${ }^{19}$ explanations for this phenomenon could be selective survival among the lower-SES areas (ie, only the healthiest individuals from low SES areas survive to old age). Previous research has shown that disease onset tends to be earlier among persons with a lower educational level, ${ }^{18}$ resulting in earlier death among low SES individuals. The results reported by Reinier $e t a l^{5}{ }^{6}$ give a hint that results similar to ours may be found in other settings. To gain further knowledge about this, our results need to be replicated in other settings.

In the studied population, the relationship between SES and OHCA incidence appeared to be stronger among men than women, when adjusted for the proportion of the population with a foreign background. However, given the limited sample size in our study after stratifying by sex, these results should be considered as preliminary, meaning that this may be a statistical artefact. The crude incidence rates followed a similar pattern among women and men, so one should exercise caution when interpreting these results.

Another patient characteristic correlated with SES is ethnicity/race. Previous research has shown that black/African American men and women have a much higher incidence of sudden cardiac death (SCD) compared with white men and women. ${ }^{20}{ }^{21}$ In a study by Zhao et al, mediation analysis suggested that $65 \%$ of this association may be the result of other patient characteristics, including income and education. ${ }^{21}$ The degree to which ethnic composition has affected our results is hard to know, but sensitivity analyses suggest that the SES relationship is present when categorising areas by the different proportions of the population with a foreign background. Such analyses are not without flaws, as the data does not allow anything other than crude analyses.

The exact mechanisms behind the SES differences in OHCA incidence are difficult to assess. Although correlated, income has been suggested to capture material living conditions, with their associated levels of psychological stress. ${ }^{22}$ Education, on the other hand, may reflect health-related knowledge and control of one's life. ${ }^{23}$ A recent study ${ }^{24}$ carried out in the Stockholm region revealed a stronger association between area-level education (vs income) and survival after OHCA. Thus, different measures of SES may yield different results. We suggest that educational efforts should be aimed at areas of lower SES, both as regards training in CPR and information on risk factors of cardiovascular disease and cardiac arrest.

Future studies should be carried out to further investigate the association found here, if possible using individual-level data. Studies on how CPR knowledge and AEDs are distributed in different socioeconomic areas could lead to a better understanding of where interventions should be aimed.

\section{Strengths and limitations}

Our study has several strengths. First, we had accurate information on the areas in which the patients lived, which has not been the case in most previous studies in the field. When studying SES differences in relation to the incidence of OHCA, it is essential to have information on the areas in which each person belongs. If the location of cardiac arrest is used, those who suffered arrest outside their homes may be categorised in the wrong area (areas with transportation hubs may show elevated incidence rates). We also used a relatively large sample size including all OHCAs in the Stockholm region over a 12-year period, and we assessed differences between relatively small areas that have been defined as population areas in other studies of health issues.
Among the limitations of this study is the fact that there was a group of patients for whom we could not find any personal information/home address. In addition, as we did not have the possibility to use individual-level SES data, we were not able to rule out the possibility of ecological fallacy. We do not have information about selective registration into the SRCR (eg, we do not know if low SES individuals are more likely or less likely to be included in the register). As a result of relatively high multicollinearity, it was difficult to investigate the separate effects of income and education.

\section{CONCLUSIONS}

Areas characterised by high SES show a significantly lower incidence of OHCA compared with those with a low SES. This relationship is not present in the older age groups $(75+)$. SES, and its interaction with age, may be an important factor to consider when planning preventive and intervention measures among high-risk populations, remembering that the effect of SES is only present in patients under 75 years of age.

\section{What is already known on this subject}

Socioeconomic status is associated with the incidence of out-of-hospital cardiac arrest.

- This relationship has been reported numerous times in North America, Asia and Europe.

\section{What this study adds}

- The relationship between socioeconomic status and out-ofhospital cardiac arrest is dependent on age.

- Among age-groups up to 75 years, there is a strong socioeconomic gradient that disappears among the oldest, suggesting a levelling effect.

Contributors MJ, PN, JH and PL designed the study. MJ performed the statistical analyses. SM and BvN provided analytical advice. MJ, MR, PN, JH and PL drafted the manuscript. All authors critically revised and edited the manuscript.

Funding The authors have not declared a specific grant for this research from any funding agency in the public, commercial or not-for-profit sectors.

Competing interests None declared.

Patient consent for publication Not required.

Data sharing statement No data are available.

Provenance and peer review Not commissioned; externally peer reviewed.

Open access This is an open access article distributed in accordance with the Creative Commons Attribution Non Commercial (CC BY-NC 4.0) license, which permits others to distribute, remix, adapt, build upon this work non-commercially, and license their derivative works on different terms, provided the original work is properly cited, appropriate credit is given, any changes made indicated, and the use is noncommercial. See: http://creativecommons.org/licenses/by-nc/4.0/.

\section{ORCID iDs}

Martin Jonsson http://orcid.org/0000-0002-3539-8317

Juho Härkönen http://orcid.org/0000-0001-9687-1932

\section{REFERENCES}

1 Kaplan GA, Keil JE. Socioeconomic factors and cardiovascular disease: a review of the literature. Circulation 1993;88:1973-98.

2 Clark AM, DesMeules M, Luo W, et al. Socioeconomic status and cardiovascular disease: risks and implications for care. Nat Rev Cardiol 2009;6:712-22. 
3 Mozaffarian D, Benjamin EJ, Go AS, et al. Heart disease and stroke statistics-2016 update: a report from the American Heart Association. Circulation 2016;133:e38-360.

4 Berdowski J, Berg RA, Tijssen JGP, et al. Global incidences of out-of-hospital cardiac arrest and survival rates: systematic review of 67 prospective studies. Resuscitation 2010;81:1479-87.

5 Reinier K, Stecker EC, Vickers C, et al. Incidence of sudden cardiac arrest is higher in areas of low socioeconomic status: a prospective two year study in a large United States community. Resuscitation 2006;70:186-92.

6 Reinier K, Thomas E, Andrusiek DL, et al. Socioeconomic status and incidence of sudden cardiac arrest. C Can Med Assoc J 2011;183:1705-12.

7 Semple HM, Cudnik MT, Sayre M, et al. Identification of high-risk communities for unattended out-of-hospital cardiac arrests using GIS. J Community Health 2013:38:277-84.

8 Ahn KO, Do SS, Hwang SS, et al. Association between deprivation status at community level and outcomes from out-of-hospital cardiac arrest: a nationwide observational study. Resuscitation 2011;82:270-6.

9 Rakun A, Allen J, Shahidah N, et al. Ethnic and neighborhood socioeconomic differences in incidence and survival from out of hospital cardiac arrest in Singapore. Prehospital Emerg Care [Internet] 2019;1-12.

10 Folke F, Gislason GH, Lippert FK, et al. Differences between out-of-hospital cardiac arrest in residential and public locations and implications for public-access defibrillation. Circulation 2010;122:623-30.

11 Castra L, Genin M, Escutnaire J, et al. Socioeconomic status and incidence of cardiac arrest: a spatial approach to social and territorial disparities. Eur J Emerg Med 2019;26:180-7.

12 Pujades-Rodriguez M, Timmis A, Stogiannis D, et al. Socioeconomic deprivation and the incidence of 12 cardiovascular diseases in 1.9 million women and men: implications for risk prediction and prevention. PLoS One 2014;9:8.

13 Straney LD, Bray JE, Beck B, et al. Are sociodemographic characteristics associated with spatial variation in the incidence of OHCA and bystander CPR rates? A population-based observational study in Victoria, Australia. BMJ Open 2016:6:e012434.

14 van Nieuwenhuizen BP, Oving I, Kunst AE, et al. Socio-economic differences in incidence, bystander cardiopulmonary resuscitation and survival from out-of-hospital cardiac arrest: a systematic review. Resuscitation 2019.

15 Tillväxt och regionplaneförvaltningen. Områdesdatabasen [Internet]. 2020. Available https://www.h6.scb.se/osdb2015/default.aspx?ReturnUrl=odb (accessed 26 Mar 2020).

16 Zeileis A, Kleiber C, Jackman S. Regression models for count data in R. J Stat Softw 2008;27:1-25.

17 Stecker EC, Reinier K, Rusinaru C, et al. Health insurance expansion and incidence of out-of-hospital cardiac arrest: a pilot study in a US metropolitan community. J Am Heart Assoc 2017;6:1-8.

18 Dupre ME. Disadvantage and age-as-leveler hypotheses educational in age-related differences patterns of disease : reconsidering the cumulative disadvantage and age-as -leveler hypotheses*. J Health Soc Behav 2007;48:1-15.

19 Ferraro KF, Farmer MM. Double jeopardy, aging as leveler, or persistent health inequality? A longitudinal analysis of white and black Americans. J Gerontol 1996;51:319-28.

20 Deo R, Safford MM, Khodneva YA, et al. Differences in risk of sudden cardiac death between blacks and whites. J Am Coll Cardiol 2018;72:2431-9.

21 Zhao D, Post WS, Blasco-Colmenares E, et al. Racial differences in sudden cardiac death atherosclerosis risk in communities study (ARIC). Circulation 2019;139:1688-97.

22 Galobardes B, Shaw M, Lawlor DA, et al. Indicators of socioeconomic position (part 1). J Epidemiol Community Heal 2006;60:7-12.

23 Mirowsky J, Ross CE. Education, learned effectiveness and health. London Rev Educ 2005:3:205-20.

24 Jonsson M, Härkönen J, Ljungman $\mathrm{P}$, et al. Survival after out-of-hospital cardiac arrest is associated with area-level socioeconomic status. Heart 2019;105:632-8. 